\title{
Violência Obstétrica: Apontamentos da Produção Científica
}

\author{
Obstetric Violence: Indications of Scientific Production
}

Violencia Obstétrica: Apuntes de la Producción Científica

\author{
Rodrigo da Silva Maia ${ }^{1}$ \\ Tereza Cristina Santos de Araújo ${ }^{2}$ \\ Eulália Maria Chaves Maia ${ }^{3}$
}

\section{Resumo}

Objetivo: o presente estudo tem por objetivo caracterizar, através de uma revisão integrativa da literatura, as produções científicas sobre a violência obstétrica contra parturientes. Método: Realizamos uma busca eletrônica por artigos, publicados até 2016, nas bases de dados MEDLINE, LILACS e SCIELO. Para a busca utilizamos a expressão: "violência obstétrica" OR "obstetric violence". Resultado: Ao todo foram encontradas 33 publicações nas bases de dados exploradas com a expressão utilizada. Após aplicação dos critérios de seleção, permaneceram um total de 15 artigos a serem escrutinados nesta revisão. Os achados sinalizam para uma concentração de publicações no ano de 2015. Há uma variedade metodológica nos estudos, com predominância de abordagens qualitativas no trato com a temática. As publicações estão vinculadas a periódicos latino-americanos. Tal dado parece sugere uma maior prevalência deste fenômeno no contexto dos países em desenvolvimento, o que pode estar relacionado ao processo recente de

${ }^{1}$ Psicólogo. Mestre em Psicologia pelo Programa de Pós-Graduação em Psicologia da Universidade Federal do Rio Grande do Norte. Autor correspondente: Av. Senador Salgado Filho, s/n, Campus Universitário, Lagoa Nova. CEP: 59078-970. Natal, Rio Grande do Norte, Brasil. E-mail: rodrigo_maia89@yahoo.com.br

${ }^{2}$ Graduanda em Psicologia pelo Centro Universitário FACEX.

${ }^{3}$ Psicóloga. Doutora em Psicologia Clínica pelo Programa de Pós-Graduação em Psicologia Clínica da Universidade de São Paulo. Docente do Departamento de Psicologia da Universidade Federal do Rio Grande do Norte.

Recebido: Out/2017 - Aceito: Dez/2017. 
reforma sanitária, de humanização nos contextos de assistência à saúde e decorrente dos altos índices e contextos de vulnerabilidade social e econômica presente nestes países. Conclusão: Os estudos revisados demonstram que a violência obstétrica é um tema complexo, que envolve aspectos como relação médico-paciente, formação em saúde, educação permanente, necessidade constante de discussão do tema humanização no cotidiano das ações e dos serviços de saúde. Sugeremse pesquisas que explorem o tema utilizando metodologias que contemplem a complexidade da temática, com o uso de métodos mistos e combinados.

\section{Descritores: Depressão; Idosos;}

\section{Envelhecimento.}

\section{Abstract}

Objective: this study aims to characterize, through an integrative literature review, the scientific productions about obstetric violence against pregnant women. Method: We conducted an electronic search for articles published until May 2016 in the databases MEDLINE, LILACS and SCIELO. For search use the expression: "violência obstétrica " OR "obstetric violence". Results: Altogether 33 publications were found in databases operated with the expression used. After applying the selection criteria, remained a total of 15 articles to be scrutinized in this review. The findings suggest a concentration of publications in 2015. There is a methodological variety in studies, especially qualitative approaches in dealing with the issue. The publications are linked to Latin American journals. This data seems to suggest a higher prevalence of this phenomenon in the context of developing countries, which may be related to the recent process of health reform, humanization in health care settings and due to the high levels and contexts of social and economic vulnerability present in these countries. Conclusion: The reviewed studies show that the obstetric violence is a complex issue, involving aspects such as doctorpatient relationship, health education, continuing education, constant need of discussion about the humanization theme in daily health's actions and services. It is suggested researches exploring the issue using methodologies that contemplate the complexity of the subject, with the use of mixed and combined methods. 
Descriptors: Parturition; Women's Health; Review.

\section{Resumen}

Objetivo: el presente estudio tiene por objetivo caracterizar, a través de una revisión integrativa de la literatura, las producciones cientificas sobre la violencia obstétrica contra parturientas. Método: Realizamos una búsqueda electrónica por artículos publicados hasta 2016 en las bases de datos MEDLINE, LILACS y SCIELO. Para la búsqueda utilizamos la expresión: "violencia obstétrica" OR "obstetric violence". Resultados: En total se encontraron 33 publicaciones en las bases de datos explotadas con la expresión utilizada. Después de la aplicación de los criterios de selección, permanecieron un total de 15 artículos a ser escrutados en esta revisión. Los hallazgos señalan para una concentración de publicaciones en el año 2015. Hay una variedad metodológica en los estudios, con predominio de abordajes cualitativos en el trato con la temática. Las publicaciones están vinculadas a periódicos latinoamericanos. Este dato parece sugerir una mayor prevalencia de este fenómeno en el contexto de los países en desarrollo, lo que puede estar relacionado con el proceso reciente de reforma sanitaria, de humanización en los contextos de asistencia a la salud y debido a los altos índices y contextos de vulnerabilidad social y económica presente en estos países. Conclusión: Los estudios revisados demuestran que la violencia obstétrica es un tema complejo, que involucra aspectos como relación médico-paciente, formación en salud, educación permanente, necesidad constante de discusión del tema humanización en el cotidiano de las acciones y de los servicios de salud. Se sugieren investigaciones que exploren el tema utilizando metodologías que contemplen la complejidad de la temática, con el uso de métodos mixtos y combinados.

\section{Descriptores: Parto; Salud de la mujer; Revisión.}

\section{Introdução}

Com a contemporaneidade, a mulher e seu processo de saúde tem ganhado visibilidade e estado no centro de discussão de políticas, programas e ações de saúde, em especial, a partir do advento da Política Nacional de Atenção Integral à Saúde da Mulher. Dentre os elementos preconizados pela política, destaca-se a ênfase à atenção e 
cuidado obstétrico, incluindo-se aqui o cuidado à assistência pré-natal e a humanização do parto $^{(1,2)}$. O parto tem ganhado o centro da discussão do cuidado obstétrico, uma vez que este é um processo singular na vida das mulheres gestantes, um momento peculiar e idiossincrático, marcado pela introdução da mulher ao papel da maternidade. Contudo, a literatura mostra que este processo tem sido vivenciado de maneira ambivalente, com percepções positivas e negativas sobre o parto e seus diferentes tipos, e comumente acompanhado de insatisfação com a assistência recebida $^{(3)}$.

Quanto a humanização do parto, a literatura ressalta que aspectos como privacidade, acompanhamento e suporte familiar, o relacionamento autêntico e a postura ética da equipe para com a parturiente e a família, práticas psicoeducativas pró-parturição - que envolvam orientações sobre o parto, estímulo ao relaxamento, por exemplo , escolha orientada pelo tipo de parto, medidas de higiene, contato mãe-filho, estímulo ao fortalecimento do laço da díade mãe-bebê e incentivo à amamentação, que em resumo sinalizam para um empoderamento feminino na parturição, são aspectos mínimos esperados durante este processo ${ }^{(4,5)}$.
Entretanto, tais características parecem estar ausentes durante $\mathrm{o}$ processo de parir. Visualiza-se a presença de pouca autonomia feminina no processo, inúmeras intervenções obstétricas invasivas e desconfortáveis, peregrinação pela procura por atendimento para o parto, bem como indicadores perinatais insatisfatórios caracterizam o cenário de violências obstétricas pelas quais tem passado as mulheres na realidade brasileira, o qual sinaliza para uma desassistência e desrespeito ao cuidado integral à mulher ${ }^{(6,7)}$.

A violência obstétrica pode caracterizar-se pela utilização de, no mínimo, uma intervenção considerada claramente prejudicial, ineficaz, desnecessária e/ou sem evidências que justifiquem o seu uso durante o parto normal ou natural. Dentre estas cita-se uso de determinadas posições, tais como a supina ou litotômica, durante o parto, infusão venosa, exame invasivos, como o retal e toques vaginais repetitivos, administração de ocitocina sem indicação fidedigna, episiotomia, clampeamento precoce do cordão, dentre outras intervenções ${ }^{(5,6,7)}$.

Além disso, destaca-se também que a literatura ressalta o lugar do trabalhador de saúde na desconsideração sobre as informações à 
parturiente no que tange as escolhas dos procedimentos. Ou seja, parece haver ausência de protagonismo e estímulo por parte dos profissionais de saúde ao empoderamento e exercício da autonomia feminino durante a parturição $^{(8)}$.

Ademais, a existência de uma representação de que o parto é algo doloroso, física e emocionalmente, e penoso gera dúvidas e apreensões nas parturientes. Tal representação parece ser reforçada pela mídia, bem como pelo paradigma biomédico em saúde, o que faz com que o parto seja percebido como requerendo procedimentos técnicos e uso de saberes especializados, o que assegura o lugar do conhecimento técnico-científico biomédico e estabelece uma relação assimétrica entre profissionais de saúde e gestante. Esta conjuntura parece sustentar a ocorrência de violências obstétricas durante o parto, ausência de protagonismo feminino e descumprimento de direitos da gestante durante o parto ${ }^{(9,10)}$.

Reconhecendo a ocorrência de episódios de desrespeito à parturiente, situações de assédios, desde físicos, emocionais e morais, contra a gestante no momento do parto, bem como a existência de abusos e ocorrências que violam os direitos obstétricos, vê-se a necessidade de se debruçar sobre o fenômeno da violência obstétrica. Diante do exposto, o presente estudo tem por objetivo caracterizar, a luz de uma revisão integrativa da literatura, as produções científicas sobre o tema da violência obstétrica contra parturientes.

\section{Método}

Este é um estudo de revisão de literatura, do tipo integrativa, elaborado a partir dos procedimentos metodológicos apresentados em Mendes, Silveira e Galvão ${ }^{(11)}$, conforme explicita o fluxograma 01 . Este tipo de estudo objetiva reunir e sintetizar achados de estudo sobre um tema ou questão específicas, aprofundando o conhecimento da temática investigada. Construiu-se o trabalho alicerçado em sugestões para qualidade de revisões de literatura ${ }^{(12,13)}$.

Após a definição da pergunta de pesquisa, procedemos a uma busca eletrônica, em junho de 2017, nas bases de dados Medical Literature Analysis and Retrieval System Online (MEDLINE), Literatura Latino Americana em Ciências da Saúde (LILACS) e Scientific Eletronic Library Online (SciELO). Realizamos a busca por artigos publicados até 2016. Para a busca utilizamos a seguinte expressão: 
"violência obstétrica" OR "obstetric violence".

Fluxograma 01. Passos da Revisão Integrativa

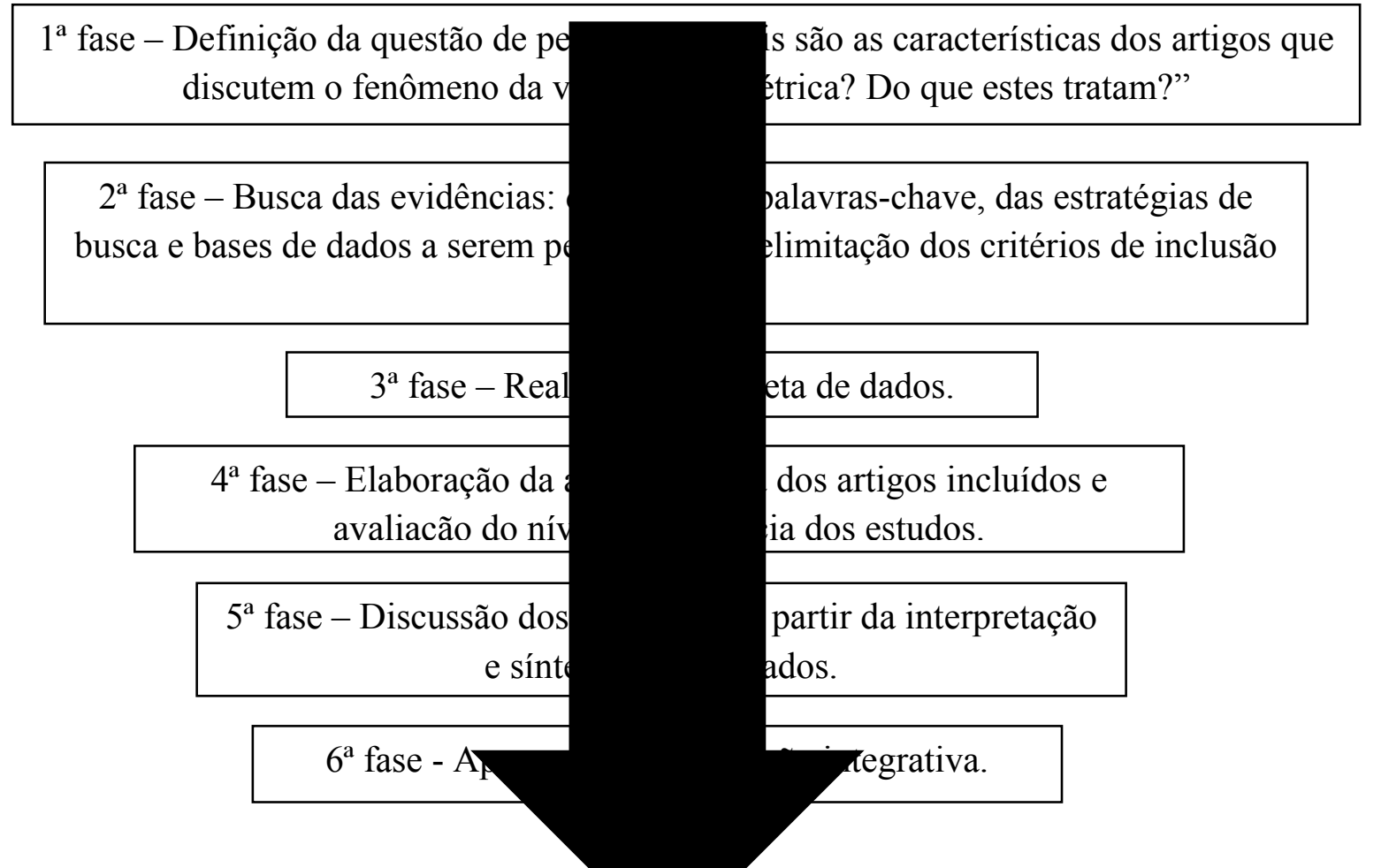

Foram incluídos no presente integra, verificando aqueles que não estudo artigos disponíveis em texto atendiam aos critérios de seleção completo, publicados nos idiomas (objetivo do estudo, inclusão e inglês, espanhol e português, e que exclusão). Os casos em que houve constassem o termo "violência obstétrica" em um dos três idiomas citados no título do trabalho, os quais foram selecionados inicialmente a partir da leitura dos títulos e resumos, quando disponíveis. Excluíram-se desta revisão editoriais, artigos de opinião, cartas ao editor, resenhas de livros, teses, dissertações e monografias.

Inicialmente, todos os títulos e resumos, quando disponíveis, foram avaliados pelo primeiro autor do estudo. Em seguida, avaliaram-se os estudos na dúvidas e/ou discordância, foram submetidos a um segundo avaliador.

A coleta das informações inspirou-se no instrumento elaborado por Coriolano-Marinus e colaboradores $^{(14)}$, o qual contemplou informações como autor, ano de publicação, periódico, objetivo, método, principais resultados. Além disso, acrescentamos a análise do nível de evidência científica. Os dados foram analisados por meio de estatística descritiva (análise bibliométrica) e 
analise de conteúdo temática. Já a avaliação do nível de evidência do artigo fora realizada com base nas proposições de Melnyk e FineoutOverholt $^{(15)}$ : I - Revisão sistemática ou meta-análise (maior nível de evidência); II - Ensaio controlado aleatório; III - Ensaio controlado sem aleatoriedade; IV - Estudo de casocontrole ou Estudo de coorte; V Revisão sistemática de estudo qualitativo ou descritivo; VI - Estudo qualitativo ou descritivo; VII - Parecer ou consenso de expertises (menor nível de evidência).

\section{Resultados e Discussão}

As características dos idosos que residem na comunidade e que são cadastrados na Estratégia Saúde da Família são apresentadas na tabela 1 . Observa-se a predominância de indivíduos que se autodeclararam pardos, com ensino fundamental incompleto e do sexo feminino. No que concerne aos principais trabalhos apresentados pelos idosos entrevistados, a maioria das mulheres desenvolvem atividade de doméstica, e os homens o serviço de pedreiro. A saber, $16 \%$ dos idosos encontram-se aposentados, 22,09\% recebem Benefício assistencial ao idoso e à pessoa com deficiência (BCP) ou Benefício de Prestação Continuada (LOAS), 5,81\% são pensionistas, no entanto, muitos desses idosos ainda continuam realizando essas e outras atividades.

Os dados da tabela 1 demonstram uma maior concentração de achados no ano de 2015 (n=6). Quanto ao periódico, visualiza-se uma distribuição heterogênea dos estudos, ressaltando-se a repetição de publicações apenas nos periódicos Revista de Obstetricia y Ginecología de Venezuela, Revista Rede de Enfermagem do Nordeste (RENE) e a Revista Venezolana de Estudios de la Mujer. Também há uma variedade metodológica nos estudos, mas os achados parecem sinalizar uma predominância de abordagens qualitativas no trato com esta temática.

Ressaltam-se algumas informações contidas no material revisado que contribuem para a compreensão do fenômeno. De modo geral, as publicações estão vinculadas a periódicos latino-americanos. Tal dado parece sinalizar para uma maior prevalência deste fenômeno no contexto dos países em desenvolvimento, o que pode ser explicado pelo processo recente de reforma sanitária e humanização da saúde no contexto destes países. 
Tabela 1. Dados Bibliométricos dos Estudos

\begin{tabular}{|c|c|c|c|c|}
\hline Autor (Ano) & Periódico & Desenho & Objetivo & Evidência \\
\hline Pozzio (2016) & Estudos Feministas & Estudo teórico & $\begin{array}{c}\text { Descrever os diferentes } \\
\text { posicionamentos em torno da } \\
\text { introdução da humanização do } \\
\text { parto. }\end{array}$ & VII \\
\hline $\begin{array}{l}\text { Andrade et al. } \\
\qquad(2016)\end{array}$ & $\begin{array}{l}\text { Rev. Brasileira de Saúde } \\
\text { Materno-Infantil }\end{array}$ & $\begin{array}{l}\text { Estudo empírico } \\
\text { transversal }\end{array}$ & $\begin{array}{l}\text { Analisar fatores relacionados a } \\
\text { ocorrência de violência obstétrica } \\
\text { decorrente da execução de } \\
\text { práticas não recomendadas ao } \\
\text { parto vaginal. }\end{array}$ & IV \\
\hline $\begin{array}{l}\text { Mc Callum; } \\
\text { Menezes; Reis } \\
\text { (2016) }\end{array}$ & $\begin{array}{l}\text { História, Ciências, } \\
\text { Saúde-Manguinhos }\end{array}$ & $\begin{array}{l}\text { Pesquisa qualitativa } \\
\text { etnográfica }\end{array}$ & $\begin{array}{c}\text { Analisa o aborto pelas } \\
\text { perspectivas de mulheres } \\
\text { internadas em uma maternidade } \\
\text { pública }\end{array}$ & VI \\
\hline $\begin{array}{l}\text { Diniz et al. } \\
\text { (2015) }\end{array}$ & $\begin{array}{l}\text { Rev. Brasileira de } \\
\text { Crescimento e } \\
\text { Desenvolvimento } \\
\text { Humano }\end{array}$ & $\begin{array}{c}\text { Pesquisa } \\
\text { bibliográfica }\end{array}$ & $\begin{array}{c}\text { Apresentar uma revisão sobre a } \\
\text { violência obstétrica. }\end{array}$ & VII \\
\hline $\begin{array}{l}\text { Regis; } \\
\text { Resende } \\
(2015)\end{array}$ & $\begin{array}{l}\text { Documentação de } \\
\text { Estudos em Linguística } \\
\text { Teórica e Aplicada } \\
\text { (DELTA) }\end{array}$ & $\begin{array}{l}\text { Pesquisa qualitativa } \\
\text { documental }\end{array}$ & $\begin{array}{l}\text { Proporcionar análise de uma carta } \\
\text { ao obstetra que tematiza sobre a } \\
\text { ocorrência de uma violência } \\
\text { obstétrica }\end{array}$ & VI \\
\hline Pickles (2015) & $\begin{array}{l}\text { The South African } \\
\text { Crime Quarterly } \\
\text { (SACQ) }\end{array}$ & Estudo teórico & $\begin{array}{l}\text { Examinar a assistência em } \\
\text { maternidade vivenciada por } \\
\text { mulheres sul-africanas. }\end{array}$ & VII \\
\hline $\begin{array}{l}\text { Silva et al. } \\
\text { (2014) }\end{array}$ & $\begin{array}{c}\text { Rev. Rede de } \\
\text { Enfermagem do } \\
\text { Nordeste (RENE) }\end{array}$ & $\begin{array}{l}\text { Relato de } \\
\text { Experiência }\end{array}$ & $\begin{array}{c}\text { Descrever a experiência de } \\
\text { enfermeiras obstetras sobre a } \\
\text { violência obstétrica. }\end{array}$ & VI \\
\hline $\begin{array}{l}\text { Sanfelice et al. } \\
\qquad(2014)\end{array}$ & $\begin{array}{c}\text { Rev. Rede de } \\
\text { Enfermagem do } \\
\text { Nordeste (RENE) }\end{array}$ & $\begin{array}{l}\text { Relato de } \\
\text { Experiência }\end{array}$ & $\begin{array}{l}\text { Relatar a experiência de } \\
\text { enfermeiras obstetras sobre a } \\
\text { transição do atendimento ao parto } \\
\text { institucionalizado para o parto em } \\
\text { domicílio. }\end{array}$ & VI \\
\hline $\begin{array}{l}\text { Terán et al. } \\
\text { (2013) }\end{array}$ & $\begin{array}{l}\text { Revista de Obstetricia y } \\
\text { Ginecología de } \\
\text { Venezuela }\end{array}$ & $\begin{array}{l}\text { Estudo empírico } \\
\text { transversal }\end{array}$ & $\begin{array}{l}\text { Avaliar a percepção de usuárias } \\
\text { sobre a atenção recebida e a } \\
\text { relação com práticas consideradas } \\
\text { violências obstétricas. }\end{array}$ & VI \\
\hline $\begin{array}{l}\text { Faneite; Feo; } \\
\text { Merlo (2012) }\end{array}$ & $\begin{array}{l}\text { Revista de Obstetricia y } \\
\text { Ginecología de } \\
\text { Venezuela }\end{array}$ & $\begin{array}{l}\text { Estudo empírico } \\
\text { transversal }\end{array}$ & $\begin{array}{c}\text { Determinar o grau de } \\
\text { conhecimento, de profissionais de } \\
\text { saúde, sobre a violência } \\
\text { obstétrica. }\end{array}$ & VI \\
\hline Cuevas (2009) & $\begin{array}{l}\text { Revista Venezolana de } \\
\text { Estudios de la Mujer }\end{array}$ & $\begin{array}{l}\text { Pesquisa qualitativa } \\
\text { baseada na } \\
\text { interpretação pós- } \\
\text { moderna feminista }\end{array}$ & $\begin{array}{l}\text { Investigar a violência obstétrica } \\
\text { com foco na medicalização e } \\
\text { patologização do processo de } \\
\text { parto de baixo risco. }\end{array}$ & VI \\
\hline
\end{tabular}

Apenas um dos artigos não é Pickles $^{(16)}$ apresenta, brevemente, a oriundo de um país latino-americano. realidade sul-africana da assistência ao 
parto, ressaltando a ocorrência de inúmeras situações de violência durante a parturição, tais como exame invasivos, administração de ocitocina sem indicação fidedigna, episiotomia, clampeamento precoce do cordão, dentre outras práticas. Neste estudo, a autora ressalta a viabilidade de transpor as experiências exitosas de humanização do parto construída em países latino americanos, como Venezuela e México, por exemplo, à África do Sul.

Pozzio $^{(17)}$, por sua vez, demonstra o processo de introdução de práticas humanizadas no processo de parturição e como médicos, e especialmente, médicas lidam com essa inovação na realidade do México. Em seu estudo, a autora ressalta que os profissionais estudados desenvolveram três tipos de posturas, a saber: posturas abertas à humanização, as intermediarias, que envolviam a necessidade de negociação de posturas colaborativas e de cuidado no processo de parto, e posturas de resistência, os quais receavam em introduzir as práticas humanizadas no seu processo de trabalho. Destaca-se que a educação continuada de profissionais de saúde pode auxiliar no processo de naturalização da humanização do parto $^{(17)}$.
Três estudos demonstraram a prevalência do fenômeno em seus estudos $^{(18-20)}$. Este primeiro estudo encontrou uma prevalência de, aproximadamente, $87 \%$ de violência obstétrica em uma população de 603 puérperas investigadas. Dentre as práticas abusivas na parturição evidenciou-se a ocorrência de esforço indevido, administração de ocitocina sem indicação clínica e uso de posição supina/litotomia ${ }^{(18)}$. Já o segundo estudo encontrou uma taxa de $26 \%$ entre 326 entrevistadas, em especial violência psicológica, como tortura e coerção, e agressões verbais, como xingamentos, os quais foram feitos pelo profissional de saúde como obstetras, enfermeiros e anestesiologistas. As puérperas parecem desconhecer mecanismos de denúncia às situações ocorridas ${ }^{(19)}$. Por fim, Téran e colaboradores ${ }^{(20)}$ estudou um total de 425 puérperas. Em seu estudo fora verificado a violência obstétrica a partir da percepção da puérpera. Destas, 67\% passaram por algum procedimento sem consentimento, $\quad 50 \%$ receberam tratamento desumanizado e um percentual de $21 \%$ das puérperas entrevistadas perceberam-se como sendo vítima de violência.

Estes dados sugerem uma naturalização de práticas abusivas no contexto da parturição, bem como uma 
medicalização do processo, em que a figura do profissional de saúde está em primeiro plano, e a parturiente é coadjuvante ao processo, fato que tem sido ressaltado por alguns estudos sobre a temática ${ }^{(21-24)}$.

Luz, Assis e Rezende ${ }^{(25)}$ também investigaram a percepção das puérperas. Contudo, neste estudo foram estudadas adolescentes, as quais foram inqueridas sobre a percepção do parto e do tratamento recebido. Foram entrevistadas 11 adolescentes com idade média de 16 anos. $\mathrm{O}$ estudo evidenciou a ocorrência de satisfação com o tratamento recebido, mas no ínterim do trabalho de parto ocorreram situações que violaram os direitos das parturientes adolescentes. A pesquisa de McCallum, Menezes e Reis ${ }^{(26)}$, por sua vez, investigou o tratamento realizado à mulher que abortou, ressaltando que a discriminação e o descaso abrolham como práticas dos profissionais de saúde diante da interrupção, voluntária ou não, da gravidez, desconsiderando o sujeito de direito que se coloca diante de seu cuidado.

Faneite, Feo e Merlo ${ }^{(27)}$ investigaram a percepção de 500 profissionais de saúde sobre o fenômeno. Este estudo verificou que os profissionais conhecem o termo violência obstétrica, já o visualizaram na prática clínica e reconhecem a existência de uma legislação que assiste e protege a mulher e os seus direitos gineco-obstétricos, mas apenas 7\% destes formalizaram a denúncia do caso, dado o qual sugere um protecionismo entre os profissionais.

$\mathrm{O}$ artigo de autoria de Sanfelice e colaboradores $^{(28)}$ apresentou a experiência de parto domiciliar a partir da perspectiva de enfermeiras. Os dados do estudo sinalizaram para uma maior satisfação ao atendimento domiciliar, pelo incentivo à humanização do parto $\mathrm{e}$ pela despatologização da parturição, e que o parto domiciliar auxilia na prevenção à violência obstétrica, a qual parece ocorrer em especial no contexto institucional. Silva e colaboradores ${ }^{(29)}$ corrobora com este achado, uma vez que em seu estudo as profissionais enfermeiras entrevistadas vivenciaram inúmeras violências obstétricas em suas rotinas de trabalho, as quais não eram denunciadas por desconhecimento de legislação e medo de represálias, por exemplo.

Por fim, o artigo de Diniz e colaboradores $^{(30)}$ apresenta uma revisão sobre a violência obstétrica, reunindo informações de literatura científica, de movimentos sociais e documentos institucionais nacionais e internacionais. $O$ estudo define os tipos de violência 
identificadas, caracterizam definições e discutem essa complexa violência, que envolve aspectos de formação dos profissionais de saúde e a organização e gestão dos serviços de saúde.

A violência obstétrica não ocorre com público ou em lugar específico. Com frequência e gravidade que abrolham exponencialmente, ela permeia os espaços de produção de saúde e de cuidado, acontece no âmbito dos serviços de saúde, e os direitos e a cidadania de quem por ela é acometido são cruelmente desrespeitados. A eminência de riscos fatais $\mathrm{e}$ consequências não fatais resultantes da violência obstétrica são notórias, Diante disto, ressalta-se a necessidade de esforços voltados à prevenção do fenômeno da violência contra a mulher, dentre as quais se inclui a violência obstétrica. Trata-se de um tema que é pauta da agenda da saúde e das políticas de estado latino-americana e em países em desenvolvimento.

\section{Conclusão}

A presente revisão concluiu que há uma variedade de métodos e técnicas no que diz respeito a investigações que envolvem o tema da violência obstétrica, com destaque para a predominância de trabalhos teóricos e de cunho qualitativo. As publicações, em sua maioria, são oriundas de periódicos latino americanos, o que sinaliza para uma maior ocorrência da violência obstétrica em países em desenvolvimento, o que pode estar relacionado ao processo recente de reforma sanitária, de humanização nos contextos de assistência à saúde e decorrente dos altos índices e contextos de vulnerabilidade social e econômica presente nestes países. Os estudos demonstram que este é um tema complexo, que envolve uma rede de elementos, tais como relação médicopaciente, formação em saúde, educação permanente, necessidade de inserção do tema humanização no cotidiano das ações e dos serviços de saúde.

O presente estudo apresenta algumas limitações, dentre as quais destacam-se as bases de dados, uma vez que estas contemplam, em sua maioria, publicações latino americanas, ibero americanas e ocidentais. Além disso, destaca-se, como alcance, a escolha pela exclusão de teses, dissertações e monografias, uma vez que estes também podem conter informações substanciais sobre a temática aportada. Por fim, sugerem-se novos estudos que integrem informações oriundas de outras fontes informacionais, para dar visibilidade as produções relativas ao tema. Ademais, 
sugerem-se pesquisas que explorem o tema, utilizando metodologias que contemplem a complexidade da temática, sugerindo-se o uso de métodos mistos e combinados, que utilizem abordagens quali-quanti no processo de construção do conhecimento acerca da violência obstétrica.

\section{Referências}

1. Costa AM, Bahia L, Conte D. A saúde da mulher e o SUS: laços e diversidades no processo de formulação, implantação e avaliação das políticas de saúde para mulheres no Brasil. Saúde (Rio de Janeiro) [periódico na internet]. 2007 [citado 2017 jul. 21]; 31(75-76-77):13-24. Disponível em: http://www.redalyc. org/pdf/4063/406345310003.pdf.

2. Santos-Neto ET, Alves KCG, Zorzal M, Lima RCD. Políticas de saúde materna no Brasil: os nexos com indicadores de saúde materno-infantil. Saúde (São Paulo) [periódico na internet]. 2008 [citado 2017 jul. 17]; 17(2):107-19. Disponível em: http://www.scielo.br/pdf/sausoc/v17n2 /11.pdf.

3. Velho MB, Santos EKA, Brüggemann $\mathrm{OM}$, Camargo $\mathrm{BV}$. Vivência do parto normal ou cesáreo: revisão integrativa sobre a percepção de mulheres. Enfermagem (Florianópolis) [periódico na internet]. 2012 [citado 2017 jun. 16]; 21(2):45866. Disponível em: http://www.sciel o.br/pdf/tce/v21n2/a26v21n2.pdf.
4. Silva RC, Soares MC, Jardim VMR, Kerber NPC, Meincke SMK. O discurso e a prática do parto humanizado de adolescentes. Enfermagem (Florianópolis) [periódico na internet]. 2013 [citado 2017 ago. 03]; 22(3):629-36. Disponível em: http://www.scielo.br/pdf/tce/v22n3/v2 2n3a08.pdf.

5. Zanardo GLP, Uribe MC, Nadal AHR, Habigzang LF. Violência obstétrica no Brasil: uma revisão narrativa. Psicologia (Belo Horizonte) [periódico na internet]. 2017 [citado 2017 set. 16]; 29(1):1-11. Disponível em: http://www.scielo.br/pdf/psoc/v29/ 1807-0310-psoc-29-e155043.pdf.

6. Leal MC, Pereira APE, Domingues RMSM, Filha MMT, Dias MAB, Nakamura-Pereira $M$, et al. Intervenções obstétricas durante $\mathrm{o}$ trabalho de parto e parto em mulheres brasileiras de risco habitual. Caderno de Saúde Pública. 2014; 30(supl 1):1732.

7. Viellas EF. Assistência pré-natal no Brasil. Caderno de Saúde Pública. 2014; 30(Supl 1): 85-100.

8. Carvalho VF, Kerber NPC, Azambuja EP, Bueno FF, Silveira RS, Barros AM. Direitos das parturientes: conhecimento da adolescente e acompanhante. Saúde (São Paulo) [periódico na internet]. 2014 [citado 2017 ago. 21]; 23(2):572-81. Disponível em: http://www.scielo.br/ pdf/sausoc/v23n2/0104-1290-sausoc23-2-0572.pdf.

9. Pereira RR, Franco SC, Baldin N. Representações sociais e decisões das gestantes sobre a parturição: protagonismo das mulheres. Saúde (São Paulo) [periódico na internet]. 2011 [citado 2017 jul. 21]: 20(3):57989. Disponível em: http://www.s cielo.br/pdf/sausoc/v20n3/05.pdf. 
10. Wolff LR, Waldow VR. Violência consentida: mulheres em trabalho de parto e parto. Saúde (São Paulo) [periódico na internet]. 2008 [citado 2017 jun. 18]; 17(3):138-51. Disponível em: http://www .scielo.br/pdf/sausoc/v17n3/14.pdf.

11. Mendes KS, Silveira RCCP, Galvão CM. Revisão integrativa: método de pesquisa para a incorporação de evidências na saúde e na enfermagem. Enfermagem (Florianópolis) [periódico na internet]. 2008 [citado 2017 jul. 02]; 17(4):75864. Disponível em: http://www .scielo.br/pdf/tce/v17n4/18.pdf.

12. Zoltowski APC, Costa AB, Teieira MAP, Koller SH. Qualidade metodológica das revisões sistemáticas em periódicos de psicologia brasileiros. Psicologia (Brasília) [periódico na internet]. 2014 [citado 2017 set. 16]; 30(1):97-104. Disponível em: http://www.scielo.br /pdf/ptp/v30n1/12.pdf.

13. Costa AB, Zoltowski APC, Koller SH, Teixeira MAP. Construção de uma escala para avaliar a qualidade metodológica de revisões sistemáticas. Saúde (Rio de Janeiro) [periódico na internet]. 2015 [citado 2017 jul. 23]; 20(8):2441-52. Disponível em: www.scielo.br/pdf/csc/v20n8/1413-81 23-csc-20-08-2441.pdf.

14.Coriolano-Marinus MWL, Queiroga BAM, Ruiz-Moreno L, Lima LS. Comunicação nas práticas em saúde: revisão integrativa da literatura. Saúde (São Paulo) [periódico na Internet]. 2014 [citado 2017 jul. 28]; 23(4):1356-69. Disponível em: http://www.scielo.br/pdf/sausoc/v23n4 /0104-1290-sausoc-23-4-1356.pdf.
15. Melnyk BM, Fineout-Overholt E. Making the case for evidence-based practice. In: Melnyk BM, FineoutOverholt E. Evidence-based practice in nursing and healthcare: a guide to best practice. Philadelphia: Lippincott Williams And Wilkins; 2005; 1: 3-24.

16. Pickles C. Eliminating abusive 'care': A criminal law response to obstetric violence in South Africa. Criminal justice (South African) [periódico na internet]. 2015 [citado 2017 set. 26]; 54(1):5-16. Disponível em: https://www.ajol.info/index.php/sa cq/article/view/127746/117274.

17. Pozzio MR. La gineco-obstetricia en México: entre el "parto humanizado" y la violencia obstetrica. Estudos Feministas (Florianópolis) [periódico na internet]. 2016 [citado 2017 ago. 10]; 24(1):101-17. Disponível em: http://www.scielo. br/pdf/ref/v24n1/1805-9584-ref-24-0100101.pdf.

18. Andrade PON, Silva JQP, Diniz CMM, Caminha MFC. Fatores associados à violência obstétrica na assistência ao parto vaginal em uma maternidade de alta complexidade em Recife, Pernambuco. Saúde Materno Infantil (Recife) [periódico na internet]. 2016 [citado 2017 jul. 19]; 16(1):29-37. Disponível em: http://www.scielo.br/pdf/rbsmi/v16n1/ 1519-3829-rbsmi-16-01-0029.pdf.

19. Pereira C, Domínguez A, Toro J. Violencia obstétrica desde la perspectiva de la paciente. Obstetrícia e Ginecologia (Caracas) [periódico na internet]. 2015 [citado 2017 jun. 23]; 75(2):81-90. Disponível em: http://www.scielo.org.ve/pdf/og/v75n2 /art02.pdf. 
20. Terán P, Castellanos C, González BM, Ramos D. Violencia obstétrica: percepción de las usuarias. Obstetrícia e Ginecologia (Caracas) [periódico na internet]. 2013 [citado 2017 ago. 29]; 73(3):171-80. Disponível em: www.scielo.org.ve/pdf/og/v73n3/art04. pdf.

21. Carneiro R. "Para chegar ao Bojador, é preciso ir além da dor": sofrimento no parto e suas potencialidades. Sexualidade, Saúde e Sociedade (Rio de Janeiro) [periódico na internet]. 2015 [citado 2017 jun. 10]; 20:91-112. Disponível em: http://www.scielo.br/pdf/sess/n20/1984 -6487-sess-20-0091.pdf.

22. Cuevas MC. Patologizando lo natural, naturalizando lo patológico improntas de la praxis obstétrica. Estudos da mulher (Caracas) [periódico na internet]. 2009 [citado 2017 ago. 11]; 14(32):147-62. Disponível em: http://www.scielo.org. e/scielo.php?script $=$ sci_arttext\&pid $=\mathrm{S}$ 1316-37012009000100011\&lng=es\& $\mathrm{nrm}=$ iso.

23. Poljak AV. La violencia obstétrica y la esterilización forzada frente al discurso médico. Estudos da mulher (Caracas) [periódico na internet]. 2009 [citado 2017 ago. 11]; 14(32):125-46. Disponível em: http://www.scielo.org. ve/scielo.php?script $=$ sci_arttext\&pid $=$ S1316-37012009000100010\&lng=es\& nrm=iso.

24. Regis JFS, Resende VM. "Daí você nasceu minha filha": análise discursiva crítica de uma carta ao obstetra. Linguística (São Paulo) [periódico na internet]. 2015 [citado 2017 out. 15]; 31(2):573-602. Disponível em: http://www.scielo.br/pdf/delta/v31n2/1 678-460X-delta-31-02-00573.pdf.
25. Luz NF, Assis TR, Rezende FR. Puérperas adolescentes: percepções relacionadas ao pré-natal e ao parto. Saúde (São Paulo) [periódico na internet]. 2015 [citado 2017 out. 15]; 40(2):80-4. Disponível em: https://www.portalnepas.org.br/abcshs/ article/view/735/676.

26. McCallum C, Menezes G, Reis AP. $\mathrm{O}$ dilema de uma prática: experiências de aborto em uma maternidade pública de Salvador, Bahia. História, Ciências e Saúde (Rio de Janeiro) [periódico na internet]. 2016 [citado 2017 jun. 16]; 23(1):37-56. Disponível em: http://www.scielo.br/pdf/hcsm/v23n1/0 104-5970-hcsm-23-1-0037.pdf.

27. Faneite J, Feo A, Merlo JT. Grado de conocimiento de violencia obstétrica por el personal de salud. Obstetrícia e Ginecologia (Caracas) [periódico na internet]. 2012 [citado 2017 jul. 23]; 72(1):4-12. Disponível em: http://www.scielo.org.ve/pdf/og /v72n1/art02.pdf.

28. Sanfelici CFO, Abbud FSF, Pregnolatto OS, Silva MG, Shimo AKK. From institutionalized birth to home birth. Enfermagem (Fortaleza) [periódico na Internet]. 2014 [citado 2017 set. 23]; 15(2):362-70. Disponível em: http://www.revistar ene.ufc.br/revista/index.php/revista/arti cle/view/1561/pdf_1.

29. Silva MG, Marcelino MC, Rodrigues LSP, Toro RC, Shimo AKK. Obstetric violence according to obstetric nurses. Enfermagem (Fortaleza) [periódico na internet]. 2014 [citado 2017 set. 23]; 15(4):7208. Disponível em: http://www.revistar ene.ufc.br/revista/index.php/revista/arti cle/view/1514/pdf_1. 
30. Diniz SG, Salgado H, Andrezzo HA, Carvalho PC, Carvalho PA, Aguiar CA, et al. Violência obstétrica como questão para a saúde pública no Brasil: origens, definições, tipologia, impactos sobre a saúde materna, e propostas para sua prevenção. Desenvolvimento Humano (São Paulo) [periódico na internet]. 2015 [citado 2017 ago. 12]; 25(3):377-84. Disponível em: http://pepsic.bvsalud. org/pdf/rbcdh/v25n3/pt_19.pdf. 